CASE REPORT
H. Shi
P. Wang
S. Wang
Q. Yu

\section{Pleomorphic Adenoma with Extensive Ossified and Calcified Degeneration: Unusual CT Findings in One Case}

SUMMARY: We report a case of pleomorphic adenoma, which presented as a large bone-attenuation mass in the parapharyngeal space. CT scanning showed a large lobulated bone-attenuation mass with a scanty soft-tissue component.
$\mathbf{P}$ leomorphic adenoma (PA), a benign tumor of the salivary glands, occurs most commonly within the parotid gland. The parapharyngeal space is a rare site for this tumor. Pathologically, PA frequently manifests with focal or partial ossified and calcified degeneration. However, total or subtotal ossified and calcified degeneration of PA is not common, and to our knowledge, the radiologic literature regarding this condition is extremely limited. In this report, we describe an unusual PA arising in the parapharyngeal space, focusing on the CT findings.

\section{Case Report}

\section{Clinical History and Physical Examination Findings}

A 70-year-old man presented with a gradually progressive painless swelling in the throat. His medical history was otherwise unremarkable. A clinical examination revealed a smooth firm bulge of the left soft palate and lateral pharyngeal wall and a diffuse firm fullness of the left retromandibular fossa. There were no pathologic lymph nodes in the neck.

\section{Radiographic Findings}

A contrast-enhanced CT scan of head and neck revealed a large lobulated bone-attenuation mass in the left parapharyngeal space, displacing the left medial pterygoid muscles and lateral pharyngeal wall. Sporadic heterogeneous low attenuations were seen, similar to those of the adjacent muscles (Fig 1A). After infusion of the contrast material, mild enhancement was seen in a portion of the low-attenuation mass (Fig 1B). A multiplanar reformatted image showed the mass with well-defined margins located in the left parapharyngeal space, extending from the base of skull to the angle of mandible (Fig 1C). There was no infiltration of the surrounding fat.

\section{Histologic Findings}

Through a mandibular swing approach to gain entry into parapharyngeal space, the tumor was excised, after controlling the left external carotid artery. On gross examination, the lesion was $7 \times 4 \times 3 \mathrm{~cm}$ with a grayish-white cut surface. Histopathologic examination showed a neoplasm having an admixture of osseous, chondroid, and epithelial components consistent with PA (Fig 1D).

Received October 10, 2007; accepted after revision November 11.

From the Department of Radiology, Ninth People's Hospital, Shanghai Jiao Tong University School of Medicine, Shanghai, China.

Please address correspondence to Shi Huimin, MD, Radiology, Shanghai Ninth People's Hospital, No. 639 Rd, Zhi Zao Ju, Shanghai 200011, China; e-mail: shihuimin@msn.com DOI 10.3174/ajnr.A0940
The postoperative course was uneventful, and there has been no evidence of recurrence for 3 years.

\section{Discussion}

PA is the most common salivary gland tumor and accounts for approximately $60 \%$ of all salivary gland neoplasms. ${ }^{1}$ The mean age at presentation is 46 years, but the age ranges from the first to the tenth decades. ${ }^{2}$ There is a slight female predominance. ${ }^{2,3}$ Approximately $80 \%$ of PAs arise in the parotid gland, $10 \%$ in the submandibular gland, and $10 \%$ in the minor salivary gland of the oral cavity, nasal cavity, and paranasal sinuses and upper respiratory and alimentary tracts. ${ }^{4,5}$

Benign PA may occur primarily in the parapharyngeal space, probably from displaced or aberrant salivary gland tissue within a lymph node. The parapharyngeal space contains mainly fatty tissue, lymphatics, and minor salivary gland tissue and is actually a potential space for tumors located lateral to the upper pharynx and extends from the skull base to the hyoid bone. This space may be the source of the minor salivary gland tumors, ${ }^{6,7}$ as it was in this case. The common clinical presentation of $\mathrm{PA}$ is a slow-growing painless mass. Therefore, a deep-seated tumor can become a large mass before it becomes symptomatic.

Histopathologically, PA shows a remarkable degree of morphology diversity. The essential components are the capsule, epithelial and myoepithelial cells, and mesenchymal or stromal elements. ${ }^{7}$ The mesenchymal-like component is mucoid/myxoid, cartilaginous, or hyalinized. Therefore, focal or partial ossified and calcified degeneration is occasionally seen in PA, but extensive ossified and calcified degeneration as seen in our cases is rare. As the outcome of the study of Harrison, ${ }^{8}$ calcification in PA was found in a lumen and in epithelial cells and consisted of needle-shaped crystals that contained calcium and phosphorus and were probably apatite; and small collections of crystals in the lumen, which were often associated with membranous cellular debris, appeared to form larger calcified masses by fusion.

In our case, CT scans showed a large lobulated bone-attenuation mass with a scanty soft-tissue component. The boneattenuation mass in CT scans has been attributed to ossification and calcification in the tumor. The sporadic lowattenuation areas were considered to be epithelial components.

The CT differential diagnosis for ossified and calcified parapharyngeal tumors is limited and includes deep-lobe parotid tumor and ectopia meningioma. In addition, if the boneattenuation mass is large, the differential diagnosis will include 

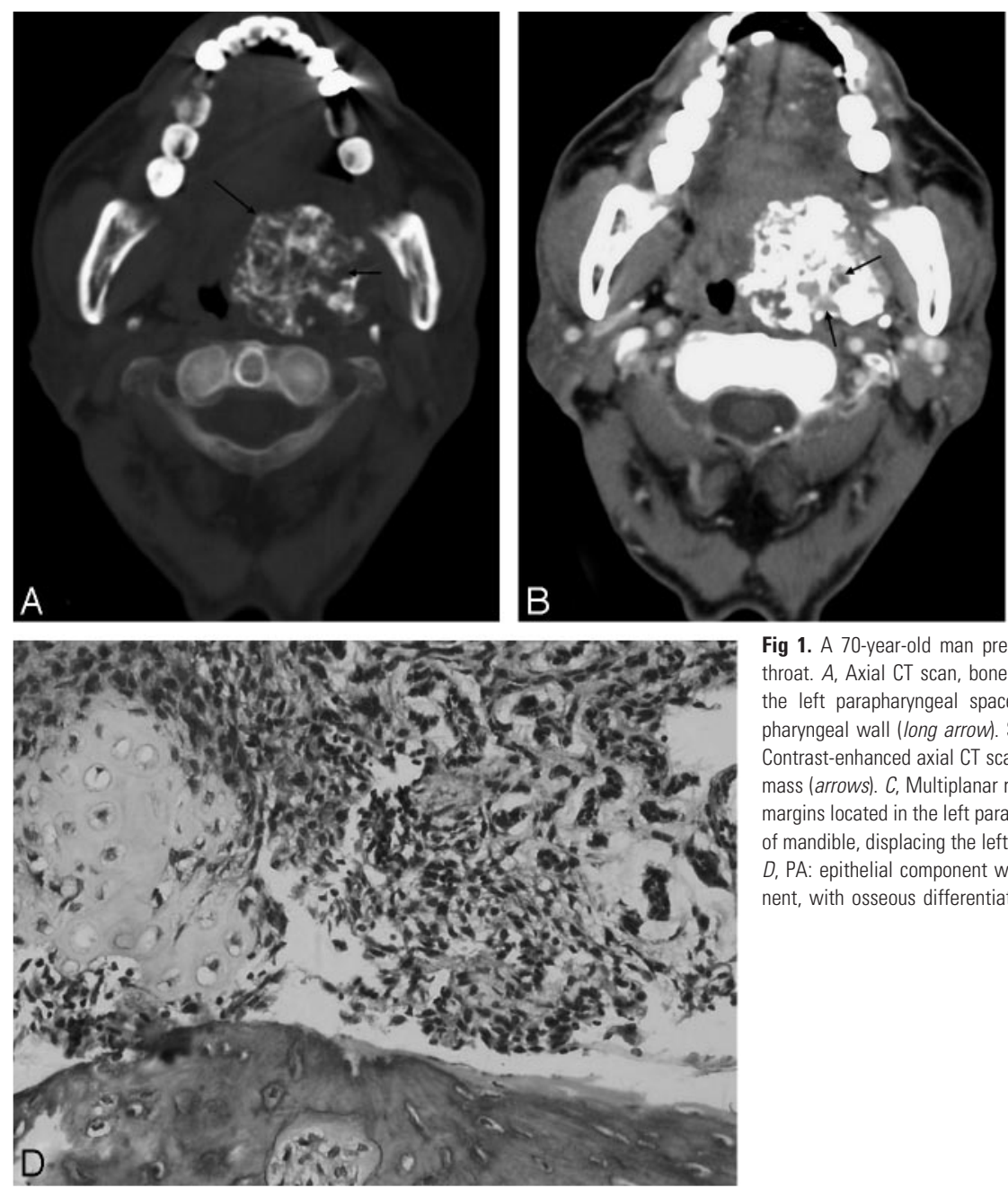

fibrous dysplasia, extraosseous osteosarcoma, and chondrosarcoma. The tumor arising de novo in the parapharyngeal space is identifiable by a fine lucent line of the compressed layer of fibroadipose tissue between the tumor and the deep lobe of the parotid gland, whereas the extension of tumor from the deep lobe of a parotid gland is not.. ${ }^{9}$ On the other hand, the ectopia meningioma can be distinguished by its accelerated and higher enhancing rate in the postinfusion CT scan. Fibrous dysplasia, extraosseous osteosarcoma, and chondrosarcoma can also be found near the parapharyngeal space. However, due to their origin from the bone, they can be identified by their attachment to their host bone.

In summary, we presented a case of benign PA arising in the parapharyngeal space with extensive ossified and calcified degeneration and its CT findings. Radiologists should be familiar with the different appearances, and this case emphasizes the wide spectrum of radiographic presentations of PA.

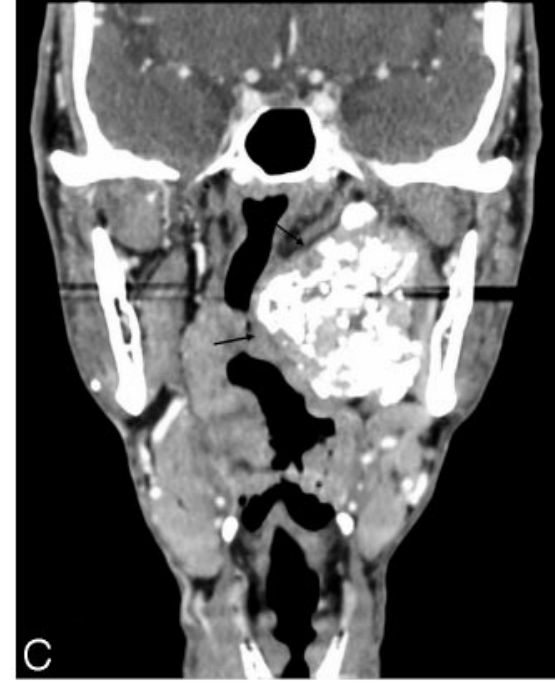

Fig 1. A 70-year-old man presented with a gradually progressive painless swelling in the throat. A, Axial CT scan, bone algorithm, shows a large lobulated bone-attenuation mass in the left parapharyngeal space, displacing the left medial pterygoid muscles and lateral pharyngeal wall (long arrow). Sporadic heterogeneous low attenuations are seen (arrow). $B$, Contrast-enhanced axial CT scan shows mild enhancement in a portion of the low-attenuation mass (arrows). C, Multiplanar reformatted coronal CT scan shows the mass with well-defined margins located in the left parapharyngeal space, extending from the base of skull to the angle of mandible, displacing the left medial pterygoid muscles and lateral pharyngeal wall (arrows). $D$, PA: epithelial component with ductal structures and a mesenchymal cartilaginous component, with osseous differentiation (hematoxylin-eosin, original magnification $\times 400$ ).

\section{References}

1. Spiro RH. Salivary neoplasms: overview of a 35 -year experience with 2,807 patients. Head Neck Surg 1986;8:177-84

2. Eveson JW, Cawson RA. Salivary gland tumours: a review of $\mathbf{2 4 1 0}$ cases with particular reference to histological types, site, age and sex distribution. J Pathol 1985;146:51-58

3. Waldron CA, el Mofty SK, Gnepp DR. Tumors of the intraoral minor salivary glands: a demographic and histologic study of $\mathbf{4 2 6}$ cases. Oral Surg Oral Med Oral Pathol 1988;66:323-33

4. Eveson JW, Cawson RA. Tumours of the minor (oropharyngeal) salivary glands: demographic study of $\mathbf{3 3 6}$ cases. J Oral Pathol 1985;14:500-09

5. Cohen MA. Pleomorphic adenoma of the cheek. Int J Oral Maxillofac Surg 1986;15:777-79

6. Ferreiro JA. Immunohistochemistry of basal cell adenoma of the major salivary glands. Histopathology 1994;24:539-42

7. Barnes L, Eveson JW, Reichart P, et al, eds. World Health Organization Classification of Tumours: Pathology and Genetics of Head and Neck Tumours. Lyon, France: IARC Press; 2005:254-58

8. Harrison JD. Ultrastructural observation of calcification in a pleomorphic adenoma of the parotid gland. Ultrastruct Pathol 1991;152:185-88

9. Heeneman H. Parapharyngeal space tumours. In: Kerr AG, ed. Scott-Brown's Otolaryngology. Vol 5. 5th ed. London, UK: Butterworth \& Co Ltd; 1987:380-91 